\title{
Mod $p$ Equality Theorem for Seiberg-Witten Invariants under $\mathbf{Z}_{p}$-actions
}

\author{
Nobuhiro NAKAMURA
}

Gakushuin University

\begin{abstract}
When a cyclic group $G$ of prime order acts on a 4-manifold $X$, we prove a formula which relates the Seiberg-Witten invariants of $X$ to those of $X / G$.
\end{abstract}

\section{Introduction}

The Seiberg-Witten invariants under group actions are investigated by many authors. In several cases, one can relate the Seiberg-Witten invariants of a 4-manifold $X$ with an action of a group $G$ to those of its quotient ( $V$-)manifold $X / G$. In fact, in the case of free actions of prime order cyclic groups $G=\mathbf{Z}_{p}$, it is proved that the Seiberg-Witten invariant of $X$ is equal modulo $p$ to a sum of invariants of $X / G$, by Ruan-Wang [11], Szymik [12] and the author [7]. This mod p equality theorem is extended to the case of double branched coverings by RuanWang [11], B. D. Park [9] and Cho-Hong [2]. On the other hand, F. Fang [3] proved a mod $p$ vanishing theorem for $\mathbf{Z}_{p}$-actions. This is extended by the author [8], and in the view point there, the $\bmod p$ vanishing theorem can be considered as a version of $\bmod p$ equality theorem: If all the involved invariants of $X / G$ are 0 by reason of negative dimensional moduli, then the invariant of $X$ is divisible by $p$.

In this paper, we shall prove a $\bmod p$ equality theorem for $\mathbf{Z}_{p}$-actions which is a generalization of both the mod $p$ equality theorem for free actions and the mod $p$ vanishing theorem. First, let us fix the notation. For an oriented closed 4-manifold $X$ with a $\operatorname{Spin}^{c}$-structure $c$, the Seiberg-Witten invariant of $(X, c)$ is denoted by $\operatorname{SW}(X, c)$, and the virtual dimension of the moduli by $d(c)$. Suppose it is given an orientation-preserving action of a finite group $G$ on $X$, and the $G$-action has a lift to $c$. In general, there are several ways of such lifts, and we use the suffix $\alpha$ to parameterize these lifts as $G_{\alpha}$. When $G=\mathbf{Z}_{p}$, we may assume $\alpha$ to be an integer satisfying $0 \leq \alpha \leq p-1$. (See $\S 3$.) When the data $\left(X, c, G_{\alpha}\right)$ of a 4-manifold $X$ with a $G$-action, a $\operatorname{Spin}^{c}$-structure $c$, and a lift $G_{\alpha}$ of the $G$-action to $c$ are given, Y. Ruan [10] defined the $G$-monopole invariant, denoted by $\operatorname{SW}\left(X, c, G_{\alpha}\right)$, which is naturally identified with the Seiberg-Witten invariant of $V$-manifold $X / G$ with a $V$-Spin ${ }^{c}$-structure $c / G_{\alpha}$. (See $\S 2$.) The virtual dimension of the moduli of $G_{\alpha}$-invariant solutions is denoted by $d\left(c, G_{\alpha}\right)$.

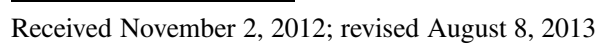


For any $G$-space $Y$, let $Y^{G}$ be the fixed point set of the $G$-action. Let $b_{\bullet}^{G}=\operatorname{dim} H_{\bullet}(X ; \mathbf{R})^{G}$, where $\bullet=1,2,+$.

Our main theorem is,

THEOREM 1.1. Let $G=\mathbf{Z}_{p}$ be the cyclic group of odd prime order $p$, and $X$ a closed oriented 4-manifold with $b_{1}=0$ and $b_{+} \geq 2$. Suppose $G$ acts on $X$ with $b_{+}^{G} \geq 2$, and the $G$-action has a lift to a $\operatorname{Spin}^{c}$-structure $c$ with $d(c)=0$. If $d\left(c, G_{\alpha}\right) \leq 0$ for any lift of the G-action, then

$$
\mathrm{SW}(X, c) \equiv \sum_{\alpha=0}^{p-1} \mathfrak{m}_{\alpha} \operatorname{SW}\left(X, c, G_{\alpha}\right) \quad \bmod p,
$$

where $\mathfrak{m}_{\alpha}$ are integers determined by the $G$-index of the Dirac operator and the $G$-action on $H^{+}(X ; \mathbf{R})$. (For instance, $\mathfrak{m}_{\alpha}=0$ if $d\left(c, G_{\alpha}\right)<0$. For the other cases, see around (3.2) for the precise definition.)

REMARK 1.3. Theorem 1.1 can be generalized to the case when $p=2$ or $b_{1}>0$ with appropriate assumptions. To avoid a complicated description, we only give the proof of the case of Theorem 1.1, and the detail of such generalizations will be left to readers. Other possibilities of generalizations will be referred in Remark 3.3 below.

The strategy of the proof of Theorem 1.1 is analogous to those in [7] and [8]. We will work out a $G$-equivariant perturbation of the monopole map. Under the $G$-action, the moduli space splits into two parts: the $G$-fixed part and the $G$-free part. When the dimension of the moduli is 0 , the number of solutions in the $G$-free part is a multiple of $p$. On the other hand, the number of $G$-invariant solutions $i s$ the $G$-monopole invariant. However, the transversality is not necessarily achieved on these $G$-invariant solutions. Then, we give a canonical way of $G$-equivariant perturbation, which enables us to determine the multiplicities of these solutions.

The organization of the paper is as follows: Section 2 gives a brief review on $G$ monopole invariants. In Section 3, we prove Theorem 1.1. In Section 4, we discuss several examples.

\section{2. $G$-monopole invariants}

In this section, we give a brief review on the $G$-monopole invariants defined by Ruan [10].

Let $X$ be a closed oriented 4-manifold, and $c$ a $\operatorname{Spin}^{c}$-structure on $X$. Let $\mathcal{G}$ be the gauge transformation group which consists of automorphisms of $c$ covering the identity map of $X$. Note $\mathcal{G}=\operatorname{Map}\left(X, S^{1}\right)$. We introduce another automorphism group $\tilde{\mathcal{G}}$ consisting of pairs $(f, \tilde{f})$, where $f: X \rightarrow X$ is an orientation preserving diffeomorphism of $X$, and $\tilde{f}: c \rightarrow c$ is an automorphism of $c$ covering $f$. Then, we have an exact sequence,

$$
1 \rightarrow \mathcal{G} \rightarrow \tilde{\mathcal{G}} \rightarrow \operatorname{Diff}^{+}(X),
$$


where $\operatorname{Diff}^{+}(X)$ is the group of orientation-preserving diffeomorphisms of $X$.

Let $G$ be a finite group. Note that giving an effective orientation-preserving $G$-action on $X$ is equivalent to giving a subgroup $G$ of $\operatorname{Diff}^{+}(X)$. Suppose such a $G$-action on $X$ is given, and $c$ satisfies $g^{*} c \cong c$ for any $g \in G$. Then the following group extension exists,

$$
1 \rightarrow \mathcal{G} \rightarrow \hat{\mathcal{G}} \stackrel{\theta}{\rightarrow} G \rightarrow 1
$$

Note that giving a lift of the $G$-action to $c$ is equivalent to giving a splitting of (2.1), that is, giving a subgroup $G_{\alpha}$ of $\hat{\mathcal{G}}$ which is isomorphic to $G$ via $\theta$.

Suppose we are given data $\left(X, c, G_{\alpha}\right)$ as above. In such a situation, Y. Ruan defined the $G$-monopole invariant [10] as follows. In this case, the Seiberg-Witten equations are $G_{\alpha^{-}}$ equivariant, and the $G_{\alpha}$-invariant moduli space $\mathcal{M}\left(X, c, G_{\alpha}\right)$ is defined as the set of equivalence classes of $G_{\alpha}$-invariant solutions modulo $G_{\alpha}$-invariant gauge transformations. The virtual dimension of $\mathcal{M}\left(X, c, G_{\alpha}\right)$ is given by

$$
d\left(c, G_{\alpha}\right)=2 \text { ind } D^{G_{\alpha}}-\left(1-b_{1}^{G}+b_{+}^{G}\right),
$$

where ind $D^{G_{\alpha}}$ is the virtual dimension of the trivial part of the $G_{\alpha}$-equivariant Dirac index. Note that we can orient all of $\mathcal{M}\left(X, c, G_{\alpha}\right)$ at the same time by fixing an orientation of $\left(H^{1}(X ; \mathbf{R}) \oplus H^{+}(X ; \mathbf{R})\right)^{G}$. If $d\left(c, G_{\alpha}\right)=0$, then the $G$-monopole invariant $\operatorname{SW}\left(X, c, G_{\alpha}\right)$ is defined as the signed count of the number of elements in $\mathcal{M}\left(X, c, G_{\alpha}\right)$. In general, we need to perturb the equations to avoid reducibles and achieve transversality. The standard argument proves that $\operatorname{SW}\left(X, c, G_{\alpha}\right)$ is well-defined if $b_{+}^{G} \geq 2$, and depends on chambers if $b_{+}^{G}=1$.

\section{Proof of Theorem 1.1}

In this section, we prove our main theorem (Theorem 1.1).

Suppose $(X, c)$ with a $G=\mathbf{Z}_{p}$-action satisfies the conditions in Theorem 1.1, and a lift of the $G$-action to $c$, say $G_{0}$, is given. Fix a $G$-invariant metric and a $G_{0}$-invariant connection $A_{0}$ on the determinant line bundle of $c$. Then the monopole map $\mu$ is a proper $G \times S^{1}$ equivariant map. Taking a finite dimensional approximation of $\mu$ [1], we have a $G \times S^{1}$ equivariant map between finite rank representations:

$$
f_{0}: V \oplus R \rightarrow W \oplus R \oplus H
$$

where $V$ and $W$ are complex representations of $G$ on which $S^{1}$ acts by multiplication, and $R$ and $H=H^{+}(X ; \mathbf{R})$ are real representations of $G$ on which $S^{1}$ acts trivially. More explicitly, when $\mathbf{C}_{j}$ is the complex 1-dimensional weight $j$ representation of $G, V$ and $W$ can be written as,

$$
\begin{gathered}
V=\mathbf{C}_{0}^{a_{0}} \oplus \mathbf{C}_{1}^{a_{1}} \oplus \cdots \mathbf{C}_{p-1}^{a_{p-1}}, \\
W=\mathbf{C}_{0}^{b_{0}} \oplus \mathbf{C}_{1}^{b_{1}} \oplus \cdots \mathbf{C}_{p-1}^{b_{p-1}} .
\end{gathered}
$$


The $G_{0}$-index of the Dirac operator associated to $A_{0}$ is written as

$$
\operatorname{ind}_{G_{0}} D_{A_{0}}=\sum_{j=0}^{p-1}\left(a_{j}-b_{j}\right) \mathbf{C}_{j} .
$$

Now, the lifts $G_{\alpha}$ are given as the splittings of the sequence

$$
1 \rightarrow S^{1} \rightarrow G \times S^{1} \rightarrow G \rightarrow 1 .
$$

(Cf. [12], Proposition 4.) The original lift $G_{0}$ corresponds to $G \times\{1\} \subset G \times S^{1}$. If we fix a generator $g$ of $G$, then the other lifts $G_{\alpha}$ are given by the subgroups generated by the elements $\left(g, e^{-2 \pi \sqrt{-1} j / p}\right) \in G \times S^{1}$ for $1 \leq j \leq p-1$. Therefore all the lifts $G_{\alpha}$ are parameterized by $\alpha=j$ where $0 \leq j \leq p-1$. As $G_{j}$-representations, $V$ and $W$ become $V \otimes \mathbf{C}_{-j}$ and $W \otimes \mathbf{C}_{-j}$.

First, perturb $f_{0} G \times S^{1}$-equivariantly so that the zero locus does not contain any reducible as follows: Take a nonzero element $v$ in $H^{+}(X ; \mathbf{R})^{G}$, and perturb $f_{0}$ to $f:=f_{0}+v$. Then $\left(f^{-1}(0)\right)^{S^{1}}=\emptyset$. (See [8], Section 2.3.)

Dividing $f$ by $S^{1}$, we obtain a section $s: B \rightarrow E$ of the vector bundle $E \rightarrow B$ which is given by

$$
\begin{gathered}
E=((V \backslash\{0\}) \times R) \times{ }_{S^{1}}(W \oplus R \oplus H), \\
B=(V \backslash\{0\}) / S^{1} \times R .
\end{gathered}
$$

When $d(c)=0, \mathrm{SW}(X, c)$ is the signed count of zero points of $s$ if $s$ is transversal to the zero section. Note that $(V \backslash\{0\}) / S^{1}$ is $G$-equivariantly homeomorphic to $P(V) \times \mathbf{R}_{+}$, where $P(V)$ is the projective space of $V$, and $\mathbf{R}_{+}$is the space of positive real numbers. The $G$-fixed point set of $P(V)$ can be written as ([8], Lemma 3.1),

$$
P(V)^{G}=\coprod_{j=0}^{p-1} P\left(\mathbf{C}_{j}^{a_{j}}\right) .
$$

Let $B_{j}=\mathbf{R}_{+} \times P\left(\mathbf{C}_{j}^{a_{j}}\right) \times R_{0}$, where $R_{0}$ is the $G$-fixed part of $R$. Then the $G$-fixed point set of $B$ decomposes into its connected components as $B^{G}=B_{0} \cup B_{1} \cup \cdots \cup B_{p-1}$.

Note that each $B_{j}$ corresponds to the lift $G_{j}$, and

$$
d\left(c, G_{j}\right)=2\left(a_{j}-b_{j}\right)-\left(1+b_{+}^{G}\right) .
$$

When $d\left(c, G_{j}\right)=0, \operatorname{SW}\left(X, c, G_{j}\right)$ is given by the signed count of zero points of $\left.s\right|_{B_{j}}$ if $\left.s\right|_{B_{j}}$ is transversal to the zero section in $E^{G}$.

Now, let us carry out the $G$-equivariant perturbation of $s$. When $d\left(c, G_{j}\right)<0$, we can perturb $s G$-equivariantly around $B_{j}$ so that $s^{-1}(0) \cap B_{j}=\emptyset$. When $d\left(c, G_{j}\right)=0$, we can 
perturb $s G$-equivariantly around $B_{j}$ so that $\left.s\right|_{B_{j}}$ is transversal along $B_{j}$. Then, the problem is how to count multiplicities of zero points on $B_{j}$.

Let $x$ be a point in $s^{-1}(0) \cap B_{j}$. We would like to describe the differentiation $(D s)_{x}$ of $s$ at $x$. The tangent space of $B$ at $x$ decomposes into the $G$-invariant direction and its complement: $T_{x} B=T_{x} B_{j} \oplus V^{\prime}$. Then $T_{x} B_{j}$ and $V^{\prime}$ can be identified as

$$
\begin{aligned}
T_{x} B_{j} & =\mathbf{R} \times \mathbf{C}_{0}^{a_{j}-1} \times R_{0}, \\
V^{\prime} & =\sum_{k \neq j} \mathbf{C}_{k-j}^{a_{k}} \oplus R^{\prime},
\end{aligned}
$$

where $R^{\prime}$ is the orthogonal complement of $R_{0}$ in $R$. By reordering $\mathbf{C}_{j}$ 's in $V^{\prime}$, rewrite $V^{\prime}$ as $V^{\prime}=\mathbf{C}_{1}^{a_{1}^{\prime}} \oplus \cdots \oplus \mathbf{C}_{p-1}^{a_{p-1}^{\prime}} \oplus R^{\prime}$, where $a_{k}^{\prime}=a_{k+j}$.

Similarly, the vertical tangent space $V_{s(x)} E$ of $E$ at $s(x)$ decomposes as, $V_{s(x)} E=W_{0} \oplus$ $W^{\prime}$, where $W_{0}$ is $G$-invariant part and $W^{\prime}$ is its complement. When we decompose $H=$ $H^{+}(X ; \mathbf{R})$ into $H_{0} \oplus H^{\prime}$, where $H_{0}$ is the $G$-fixed part and $H^{\prime}$ its complement, $W^{\prime}$ can be identified with

$$
W^{\prime}=\sum_{k \neq j} \mathbf{C}_{k-j}^{b_{j}} \oplus R^{\prime} \oplus H^{\prime} .
$$

Let us choose orientations of $H$ and $H_{0}$ (hence $H^{\prime}$ too), and fix an arbitrary identification $H^{\prime}=\mathbf{C}_{1}^{h_{1}} \oplus \cdots \oplus \mathbf{C}_{p-1}^{h_{p-1}}$ so that $H=H_{0} \oplus H^{\prime}$ and $H_{0} \oplus \mathbf{C}_{1}^{h_{1}} \oplus \cdots \oplus \mathbf{C}_{p-1}^{h_{p-1}}$ have same orientation. (Here, we used the assumption that $p$ is odd.) Rewrite $W^{\prime}$ as $W^{\prime}=\mathbf{C}_{1}^{b_{1}^{\prime}} \oplus \cdots \oplus \mathbf{C}_{p-1}^{b_{p-1}^{\prime}} \oplus R^{\prime}$, where $b_{k}^{\prime}=b_{k-j}+h_{k}$. Let $L_{0}$ be the linear map which is the composition of the following maps:

$$
L_{0}: V^{\prime} \stackrel{D s_{x}}{\longrightarrow} T_{s(x)} E \stackrel{p_{v}}{\longrightarrow} V_{s(x)} E \stackrel{p_{w}}{\longrightarrow} W^{\prime},
$$

where $p_{v}$ and $p_{w}$ are the orthogonal projections.

We will cancel out common parts in $V^{\prime}$ and $W^{\prime}$ by a perturbation by a $G$-linear map. We give a local model of this as follows. Let $e_{k}=\min \left\{a_{k}^{\prime}, b_{k}^{\prime}\right\}$. We can take an orientationpreserving $G$-linear map $l: V^{\prime} \rightarrow W^{\prime}$ so that $\operatorname{Im}\left(L_{0}+l\right) \cong \sum_{k} \mathbf{C}_{k}^{e_{k}} \oplus R^{\prime}$. Let $W_{e}=\operatorname{Im}\left(L_{0}+l\right)$ and its complement in $W^{\prime}$ be $W_{r}$, and $V_{r}=\operatorname{Ker}\left(L_{0}+l\right)$ and its complement in $V^{\prime}$ be $V_{e}$. Then,

$$
\begin{gathered}
V^{\prime}=V_{e} \oplus V_{r}, \\
W^{\prime}=W_{e} \oplus W_{r}, \\
V_{e} \cong W_{e} \cong \sum_{k} \mathbf{C}_{k}^{e_{k}} \oplus R^{\prime} .
\end{gathered}
$$

Next, we give a local model of perturbation in the direction of $V_{r}$. Let

$$
I=\left\{k \mid m_{k}=a_{k}^{\prime}-e_{k}>0\right\} \text { and } I^{\prime}=\left\{k \mid n_{k}=b_{k}^{\prime}-e_{k}>0\right\} .
$$


Then

$$
V_{r}=\sum_{k \in I} \mathbf{C}_{k}^{m_{k}}, \quad W_{r}=\sum_{k \in I^{\prime}} \mathbf{C}_{k}^{n_{k}} .
$$

Note that $I \cap I^{\prime}=\emptyset$ and $\operatorname{dim} V_{r}=\operatorname{dim} W_{r}$. We will perturb $s$ around $x$ by a (nonlinear) $G$-equivariant map $\psi: V_{r} \rightarrow W_{r}$. The next example will illustrate how to take $\psi$.

EXAmPle 3.1. Suppose $G=\mathbf{Z}_{5}, V_{r}=\mathbf{C}_{1} \oplus \mathbf{C}_{4}$ and $W_{r}=\mathbf{C}_{2} \oplus \mathbf{C}_{3}$. Then take $\psi: \mathbf{C}_{1} \oplus \mathbf{C}_{4} \rightarrow \mathbf{C}_{2} \oplus \mathbf{C}_{3}$ which is given by $\psi(z, w)=\left(z^{2}, w^{2}\right)$. If we perturb $s$ around $x$ by $\psi$, then the multiplicity of $x$ is equal modulo 5 to $2 \times 2=4$. As another choice, we can take $\psi$ given by $\psi(z, w)=\left(w^{3}, z^{3}\right)$. In this case, the multiplicity of $x$ is also equal modulo 5 to $3 \times 3 \equiv 4$. The multiplicity 4 can be calculated by $2 \cdot 3 / 1 \cdot 4 \equiv 4$ in the finite field $\mathbf{F}_{5}$.

The general case is given as follows. Let $\left(z_{1}, \ldots, z_{r}\right)$ be the coordinate of $V_{r}$ where $z_{k} \in \mathbf{C}_{i_{k}}$, and $\left(w_{1}, \ldots, w_{r}\right)$ be that of $W_{r}$ where $w_{k} \in \mathbf{C}_{i_{k}^{\prime}}$. Then $\psi: V_{r} \rightarrow W_{r}$ is given by

$$
\psi\left(z_{1}, \ldots, z_{r}\right)=\left(z_{1}^{i_{1}^{\prime} / i_{1}}, \ldots, z_{r}^{i_{r}^{\prime} / i_{r}}\right),
$$

where $i_{k}^{\prime} / i_{k}$ is calculated in $\mathbf{F}_{p}$, and identified with an integer which represents it.

The multiplicity $\mathfrak{m}_{j}$ of $x$ is given by

$$
\mathfrak{m}_{j}=\frac{\prod_{k=1}^{r} i_{k}^{\prime}}{\prod_{k=1}^{r} i_{k}} .
$$

By using an appropriate $G$-invariant cut-off function, perturb the section $s$ around $x$ by $l+\psi$. For every point in $s^{-1}(0) \cap B^{G}$, such a perturbation should be carried out. We also need to perturb $s G$-equivariantly on the free part $B \backslash B^{G}$. This is easy.

Now, we complete the proof of Theorem 1.1. By the perturbation so far, each of zeros of $s$ on $B^{G}$ has its multiplicity $\mathfrak{m}_{j}$. On the other hand, $G=\mathbf{Z}_{p}$ acts freely on $s^{-1}(0) \cap\left(B \backslash B^{G}\right)$. Hence, the relation (1.2) holds.

REMARK 3.3. In the proof above, the assumption $d(c)=0$ is not essential. In the case when $d(c)>0$, we can use the technique of cutting down the moduli space as in §3(iii) in [8]. On the other hand, the assumption $d\left(c, G_{\alpha}\right) \leq 0$ seems essential to our proof. It would be an interesting problem to consider the case when $d\left(c, G_{\alpha}\right)>0$.

REMARK 3.4. Another possibility of generalization is to consider $p$-fold branched coverings. As mentioned in $\S 1$, the case of 2 -fold branched covering is studied by $[11,9,2]$ One could try to prove similar results for higher orders.

\section{Examples}

In this section, we give several examples. 
4.1. Example 1. Let $X$ be the $K 3$ surface of the Fermat type in $\mathbf{C P}^{3}$ defined by the equation $z_{0}^{4}+z_{1}^{4}+z_{2}^{4}+z_{3}^{4}=0$. Let $G=\mathbf{Z}_{3}$ act on $X$ by permutation of components. Let $c$ be the $\operatorname{Spin}^{c}$-structure determined by the spin structure, and consider the lift $G_{0}$ of the $G$-action to $c$ whose induced action on the determinant line bundle is just the diagonal action $X \times \mathbf{C}_{0}$. Then, the $G_{0}$-index of the Dirac operator is written as $\operatorname{ind}_{G_{0}} D=2 \mathbf{C}_{0}$. (See [5].) The finite dimensional approximation of the monopole map has the form,

$$
f: \mathbf{C}_{0}^{x+2} \oplus \mathbf{C}_{1}^{y} \oplus \mathbf{C}_{2}^{z} \rightarrow \mathbf{C}_{0}^{x} \oplus \mathbf{C}_{1}^{y} \oplus \mathbf{C}_{2}^{z} \oplus \mathbf{R}^{3},
$$

where $\mathbf{R}$ is the real 1-dimensional trivial representation. It follows that $d\left(c, G_{0}\right)=0$, $d\left(c, G_{1}\right)=d\left(c, G_{2}\right)<0$, and, by Theorem 1.1 ,

$$
\mathrm{SW}(X, c) \equiv \mathrm{SW}\left(X, c, G_{0}\right) \bmod 3 .
$$

In fact, $\mathrm{SW}(X, c)=\mathrm{SW}\left(X, c, G_{0}\right)=1$, because there exists the unique $G$-invariant solution with constant spinor by the perturbation by a $G$-invariant holomorphic 2 -form. We remark that the action in Proposition 4.11 of [6] gives a similar example in the case of $G=\mathbf{Z}_{5}$.

4.2. Example 2. D.-Q. Zhang introduced a holomorphic $G=\mathbf{Z}_{3}$-action on a $K 3$ surface $X$ with $b_{+}^{G}=1$ ([14], Example 5.3, due to S. Tsunoda). Let $c$ be the spin, and consider the lift $G_{0}$ as in $\S 4.1$. In this case, the finite dimensional approximation is of the form,

$$
f: \mathbf{C}_{0}^{x} \oplus \mathbf{C}_{1}^{y+1} \oplus \mathbf{C}_{2}^{z+1} \rightarrow \mathbf{C}_{0}^{x} \oplus \mathbf{C}_{1}^{y} \oplus \mathbf{C}_{2}^{z} \oplus \mathbf{R} \oplus \mathbf{C}_{1} .
$$

Then, $d\left(c, G_{0}\right)<0, d\left(c, G_{1}\right)=d\left(c, G_{2}\right)=0$. Note that $b_{+}^{G}=1$ in this case, and therefore $\mathrm{SW}\left(X, c, G_{\alpha}\right)$ depend on chambers. Nevertheless, the formula (1.2) in Theorem 1.1 holds for any chamber as

$$
\mathrm{SW}(X, c) \equiv \mathrm{SW}\left(X, c, G_{1}\right)+2 \mathrm{SW}\left(X, c, G_{2}\right) \bmod 3 .
$$

In fact, the following occurs:

Proposition 4.1. In a chamber $\mathcal{C}_{+}, \mathrm{SW}\left(X, c, G_{1}\right)=1$ and $\mathrm{SW}\left(X, c, G_{2}\right)=0$. In another chamber $\mathcal{C}_{-}, \operatorname{SW}\left(X, c, G_{1}\right)=0$ and $\operatorname{SW}\left(X, c, G_{2}\right)=-1$.

REMARK 4.2. In the chamber $\mathcal{C}_{+}$, the formula (1.2) holds as $1 \equiv 1+2 \cdot 0$. On the other hand, in $\mathcal{C}_{-}$, the formula (1.2) holds as $1 \equiv 0+2 \cdot(-1)$.

To prove Proposition 4.1, we note the next.

LEMMA 4.3. X admits a Kähler form $\omega$ preserved by the $G$-action.

Proof. Let us recall the construction of the $\log$ Enriques surface $\bar{S}=X / G$ ([14], Example 5.3). Let $x, y, z$ be the homogeneous coordinates of $\mathbf{C P}^{2}$. Consider three cuspidal cubic curves in $\mathbf{C P}^{2}$ :

$$
C_{1}: x^{3}=y^{2} z, \quad C_{2}: y^{3}=z^{2} x, \quad C_{3}: z^{3}=x^{2} y .
$$


Let $\xi$ be a primitive 7 th root of the unity. Then $C_{1} \cap C_{2} \cap C_{3}=\left\{\left(\xi^{i}: \xi: 1\right) \mid 0 \leq i \leq 6\right\}$. Let $\tau: S \rightarrow \mathbf{C P}^{2}$ be the blowing up of cusps $(1: 0: 0),(0: 1: 0),(0: 0: 1)$, and 7 points in $C_{1} \cap C_{2} \cap C_{3}$. Then $S$ contains three disjoint nonsingular (-3)-curves from $C_{1}, C_{2}$ and $C_{3}$. Collapsing these $(-3)$-curves, we obtain the surface $\bar{S}$ whose covering is a $K 3$. These surfaces fit into the following diagram

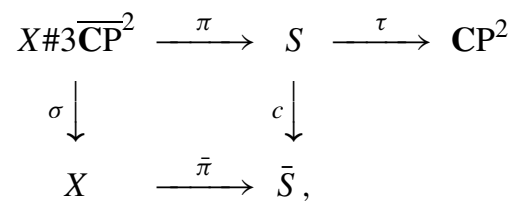

where $\sigma$ and $\tau$ are blowing up, $c$ is the collapsing map, $\pi$ is a $G$-fold covering branched along the $(-3)$-spheres, and $\bar{\pi}$ is a $G$-cover. Note that $X \# 3 \overline{\mathbf{C P}}^{2}$ has a $G$-invariant Kähler form obtained by pulling back a Kähler form on $\mathrm{CP}^{2}$ via $\tau$ and $\pi$. By blowing down, we have a Kähler form $\omega$ on $X$ which is preserved by the $G$-action.

Proof of Proposition 4.1. The positive spinor bundle $S^{+}$of $c$ can be written as $S^{+}=I \oplus K_{X}^{-1}$, where $I$ is a trivial bundle and $K_{X}$ is the canonical line bundle of $X$ (which is also trivial). Therefore, a spinor $\phi$ has two components $\phi=(\alpha, \beta)$. Since the $G$-action on $K_{X}^{-1}$ is given by $X \times \mathbf{C}_{2}$ and we fix the lift $G_{0}$ so that $\operatorname{det} S^{+}=I \otimes K_{X}^{-1}=X \times \mathbf{C}_{0}$, the $G_{0^{-}}$ action on $I$ is given by $I=X \times \mathbf{C}_{1}$. By Taubes' perturbation [4](cf. [13]) adding -ir $\omega$, we have a unique solution such that $\alpha=$ const. and $\beta=0$. This solution is $G_{1}$-invariant. On the other hand, if we use the perturbation adding $+i r \omega$, then the roles of $\alpha$ and $\beta$ are exchanged. Therefore, we have a unique solution with $\alpha=0$ and $\beta=$ const. which is $G_{2}$-invariant. These two belong to different chambers. By considering the orientations readily, the proof is completed.

REMARK 4.4. In this case, the formula $\operatorname{SW}(X, c)=\operatorname{SW}\left(X, c, G_{1}\right)-\operatorname{SW}\left(X, c, G_{2}\right)$ holds. In fact, the perturbation adding $+i r \omega$ corresponds to a linear but orientation-reversing perturbation by $\psi: \mathbf{C}_{1} \rightarrow \mathbf{C}_{2}$ given by $\psi(z)=\bar{z}$.

Several actions of higher order $G$ in [14] give similar examples.

\section{References}

[ 1 ] S. BAuer and M. Furuta, A stable cohomotopy refinement of Seiberg-Witten invariants: I, Invent. Math. 155 (2004), 1-19.

[2] Y. S. Cho and Y. H. Hong, Seiberg-Witten invariants and (anti-)symplectic involutions, Glasgow Math. J. 45 (2003), 401-413.

[ 3 ] F. FANG, Smooth group actions on 4-manifolds and Seiberg-Witten invariants, Internat. J. Math. 9, No. 8 (1998), 957-973.

[ 4 ] D. Kotschick, The Seiberg-Witten invariants of symplectic four-manifolds (after C. H. Taubes), Séminaire Bourbaki, Vol. 1995/96. Astérisque 241 (1997), Exp. No. 812, 4, 195-220.

[ 5 ] X. LiU and N. NAKAmura, Pseudofree Z/3-actions on $K 3$ surfaces, Proc. Amer. Math. Soc. 135 (2007), no. 3, 903-910. 
[ 6 ] X. LiU and N. NAKAmURA, Nonsmoothable group actions on elliptic surfaces, Topology Appl. 155 (2008), no. 9, pp 946-964.

[ 7 ] N. Nakamura, A free $\mathbf{Z}_{p}$-action and the Seiberg-Witten invariants, J. Korean Math. Soc. 39 (2002), No. 1, 103-117.

[ 8 ] N. Nakamura, Mod $p$ vanishing theorem of Seiberg-Witten invariants for 4-manifolds with $\mathbf{Z}_{p}$-actions, Asian J. Math. 10 (2006), no. 4, 731-748.

[ 9 ] B. D. PARK, Seiberg-Witten invariants and branched covers along tori, Proc. Amer. Math. Soc. 133 (2005), no. 9, 2795-2803.

[10] Y. RUAN, Virtual neighborhoods and the monopole equations, Topics in symplectic 4-manifolds (Irvine, CA, 1996), 101-116, First Int. Press Lect. Ser., I, Int. Press, Cambridge, MA, 1998.

[11] Y. RUAN and S. WANG, Seiberg-Witten invariants and double covers of 4-manifolds, Comm. Anal. Geom. 8 (2000), No. 3, 477-515.

[12] M. SzYMIK, Bauer-Furuta invariants and Galois symmetries, Q. J. Math. 63 (2012), no. 4, 1033-1054.

[13] C. H. TAubes, $\mathrm{SW} \Rightarrow$ Gr: from the Seiberg-Witten equations to pseudo-holomorphic curves, J. Amer. Math. Soc. 9 (1996), no. 3, 845-918.

[14] D.-Q. ZhANG, Logarithmic Enriques surface, J. Math. Kyoto Univ. 31 (1991), 419-466.

\author{
Present Address: \\ Department of Mathematics, \\ GAKUSHUIN UNIVERSITY, \\ 1-5-1, MeJiro, TOSHIMA-KU, TOKYO, 171-8588 JAPAN. \\ e-mail: nobuhiro@math.gakushuin.ac.jp
}

